\title{
Pathway Fractional Integral Formulas Involving $\delta$-Function in the Kernel
}

\author{
Hafte Amsalu (D), Biniyam Shimelis $(\mathbb{D}$, and D. L. Suthar $(\mathbb{D}$ \\ Department of Mathematics, Wollo University, P.O. Box. 1145, Dessie, Ethiopia \\ Correspondence should be addressed to D. L. Suthar; dlsuthar@gmail.com
}

Received 24 April 2020; Revised 8 May 2020; Accepted 15 May 2020; Published 20 June 2020

Guest Editor: Praveen Agarwal

Copyright (C) 2020 Hafte Amsalu et al. This is an open access article distributed under the Creative Commons Attribution License, which permits unrestricted use, distribution, and reproduction in any medium, provided the original work is properly cited.

In this paper, we present several composition formulae of pathway fractional integral operators connected with $\mathcal{S}$-function. Here, we point out important links to known outcomes for some specific cases with our key results.

\section{Introduction and Preliminaries}

In recent years, fractional calculus has become a significant instrument for the modeling analysis and plays a significant role in different fields, for example, material science, science, mechanics, power, economy, and control theory. In addition, a number of researchers have investigated a variety of fractional calculus operators in the depth level of properties, implementation methods, and complex modifications. Other analogous topics are also very active and extensive around the world. One may refer to the research monographs in $[1,2]$.
$\mathcal{S}$-function. Recently, Saxena and Daiya [3] defined and studied a special function called as $\mathcal{S}$-function (also see [4]) and its relation with other special functions, which include generalized $\mathscr{K}$-function, $\mathscr{M}$-series, $k$-Mittag-Leffler function, Mittag-Leffler type functions, and other many special functions. These special functions have recently found essential applications in solving problems in applied sciences, biology, physics, and engineering.

The $\mathcal{S}$-function is defined for $\sigma, \eta, \varepsilon, \tau \in \mathbb{C}, \mathfrak{R}(\sigma)>0$, $k \in \Re, \quad \Re(\sigma)>k \Re(\tau), \quad l_{i}(i=1,2,3, \ldots, p)$, $m_{j}(j=1,2,3, \ldots, q)$, and $p<q+1$ as

$$
\mathcal{S}_{(p, q)}^{\sigma, \eta, \varepsilon, \tau, k}\left[l_{1}, l_{2}, \ldots, l_{p} ; m_{1}, m_{2}, \ldots, m_{q} ; x\right]=\sum_{n=0}^{\infty} \frac{\left(l_{1}\right)_{n} \ldots\left(l_{p}\right)_{n}(\varepsilon)_{n \tau, k}}{\left(m_{1}\right)_{n} \ldots\left(m_{q}\right)_{n} \Gamma_{k}(n \sigma+\eta)} \frac{x^{n}}{n !},
$$

Here, $k$-Pochhammer symbol is as follows:

$$
(\varepsilon)_{n, k}= \begin{cases}\frac{\Gamma_{k}(\varepsilon+n k)}{\Gamma_{k}(\varepsilon)}, & (k \in \Re, \varepsilon \in \mathbb{C} /\{0\}), \\ \varepsilon(\varepsilon+k) \ldots(\varepsilon+(n-1) k), & (n \in \mathbb{N}, \varepsilon \in \mathbb{C}) .\end{cases}
$$

Also, the $k$-gamma function is

$$
\Gamma_{k}(\varepsilon)=k^{(\varepsilon / k)-1} \Gamma\left(\frac{\varepsilon}{k}\right)
$$

where $\varepsilon \in \mathbb{C}, k \in \Re$, and $n \in \mathbb{N}$, introduced by Díaz and Pariguan [5] (see also Romero and Cerutti [6]).

Several major special cases of the $\delta$-function are described as follows:

(i) For $p=q=0$, the generalized $k$-Mittag-Leffler function from Saxena et al. [7] (see $[8,9]$ ) is 


$$
\mathscr{E}_{k, \sigma, \eta}^{\mathcal{\varepsilon}, \tau}(x)=\mathcal{S}_{(0,0)}^{\sigma, \eta, \mathcal{\varepsilon}, \tau, k}[-;-; x]=\sum_{n=0}^{\infty} \frac{(\varepsilon)_{n \tau, k}}{\Gamma_{k}(n \sigma+\eta)} \frac{x^{n}}{n !}, \mathfrak{R}\left(\frac{\sigma}{k}-\tau\right)>p-q
$$

(ii) For $k=\tau=1$, the $\mathcal{S}$-function is the generalized $\mathscr{K}$-function, introduced by Sharma [10] (see also [11]):

(4)

$$
\begin{aligned}
\mathscr{K}_{(p, q)}^{\sigma, \eta, \varepsilon}\left[l_{1}, \ldots, l_{p} ; m_{1}, \ldots, m_{q} ; x\right] & =\mathcal{S}_{(p, q)}^{\sigma, \eta, \varepsilon, 1,1}\left[l_{1}, \ldots, l_{p} ; m_{1}, \ldots, m_{q} ; x\right] \\
& =\sum_{n=0}^{\infty} \frac{\left(l_{1}\right)_{n} \ldots\left(l_{p}\right)_{n}(\varepsilon)_{n}}{\left(m_{1}\right)_{n} \ldots\left(m_{q}\right)_{n} \Gamma(n \sigma+\eta)} \frac{x^{n}}{n !}, \quad \mathfrak{R}(\sigma)>p-q .
\end{aligned}
$$

(iii) For $\tau=k=\varepsilon=1$, the $\mathcal{S}$-function reduced to generalized $\mathscr{M}$-series introduced by Sharma and Jain [12](detail [13]) is

$$
\begin{aligned}
\mathscr{M}_{(p, q)}^{\sigma, \eta}\left[l_{1}, \ldots, l_{p} ; m_{1}, \ldots, m_{q} ; x\right] & =\mathcal{S}_{(p, q)}^{\sigma, \eta, 1,1,1}\left[l_{1}, \ldots, l_{p} ; m_{1}, \ldots, m_{q} ; x\right] \\
& =\sum_{n=0}^{\infty} \frac{\left(l_{1}\right)_{n} \ldots\left(l_{p}\right)_{n} x^{n}}{\left(m_{1}\right)_{n} \ldots\left(m_{q}\right)_{n} \Gamma(n \sigma+\eta)}, \quad \mathfrak{R}(\sigma)>p-q-1 .
\end{aligned}
$$

Recently, an expending pathway fractional integral (PFI) operator introduced by Nair [14], which was earlier defined by Mathai [15] and Mathai and Haubold [16, 17], is defined as follows:

$$
\left(\mathscr{P}_{0+}^{\lambda, \varsigma} f\right)(x)=x^{\lambda} \int_{0}^{[x /(a(1-\varsigma))]}\left(1-\frac{a(1-\varsigma) \xi}{x}\right)^{\lambda /(1-\varsigma)} f(\xi) \mathrm{d} \xi
$$

where Lebesgue measurable function $f \in \mathscr{L}(a, b)$ for real or complex term valued function, $\lambda \in \mathbb{C}, \mathfrak{R}(\lambda)>0, a>0$, and $\varsigma<1$ ( $\varsigma$ is a pathway parameter).
The pathway model for a real scalar $\varsigma$ and scalar random variables is represented by the probability density function (p.d.f.) in the following manner:

$$
f(x)=\frac{c}{|x|^{1-v}}\left[1-a(1-\varsigma)|x|^{\rho}\right]^{\lambda /(1-\varsigma)},
$$

where $\quad x \in(-\infty, \infty) ; \lambda>0 ; \rho>0 ;\left[1-a(1-\varsigma)|x|^{\rho}\right]^{\lambda /(1-\varsigma)}>0$; $v>0$ and $\varsigma$ and $c$ denote the pathway parameter and normalizing constant, respectively.

Additionally, for $\varsigma \in \mathfrak{R}$, the normalizing constants are expressed in the following way:

$$
c= \begin{cases}\frac{1}{2} \frac{\rho[a(1-\varsigma)]^{v / \rho} \Gamma(v / \rho+\lambda /(1-\varsigma)+1)}{\Gamma(v / \rho) \Gamma(\lambda /(1-\varsigma)+1)}, & (\varsigma<1), \\ \frac{1}{2} \frac{\rho[a(1-\varsigma)]^{v / \rho} \Gamma(\lambda /(\varsigma-1))}{\Gamma(v / \rho) \Gamma(\lambda /(\varsigma-1)-v / \rho)}, & \left(\frac{1}{\varsigma-1}-\frac{v}{\rho}>0, \varsigma>1\right), \\ \frac{1}{2} \frac{[a \lambda]^{v / \rho}}{\Gamma(v / \rho)}, & (\varsigma \longrightarrow 1) .\end{cases}
$$

It is noted that if $\varsigma<1$, finite range density with $\left[1-a(1-\varsigma)|x|^{\rho}\right]^{\lambda /(1-\varsigma)}>0$ and (8) can be considered a member of the extended generalized type- 1 beta family. Also, the triangular density, the uniform density, the extended type-1 beta density and various other probability density functions are precise special cases of the pathway density function defined in (8) for $\varsigma<1$.

For example, if $\varsigma>1$ and by setting $(1-\varsigma)=-(\varsigma-1)$ in (7), then we have 


$$
\begin{aligned}
\left(\mathscr{P}_{0+}^{\lambda, \varsigma} f\right)(x) & =x^{\lambda} \int_{0}^{[x /-a(\varsigma-1)]}\left(1+\frac{a(\varsigma-1) \xi}{x}\right)^{\lambda /-(\varsigma-1)} f(\xi) \mathrm{d} \xi \\
f(x) & =\frac{c}{|x|^{1-v}}\left[1+a(\varsigma-1)|x|^{\rho}\right]^{\lambda /-(\varsigma-1)}
\end{aligned}
$$

provided that $x \in(-\infty, \infty) ; \rho>0 ; \lambda>0$; and $\varsigma>1$ characterize the extended generalized type- 2 beta model for real $x$. The specific cases of density function (11) include the type-2 beta density function, the $p$ density function, and the Student's $t$ density function. For $\varsigma \longrightarrow 1,(7)$ diminishes to the Laplace integral transform.

In a similar way, if $\varsigma=0, a=1$, and $\lambda$ takes the place of $\lambda-1$, then (7) diminishes to the familiar Riemann-Liouville (R-L) fractional integral operator $\mathscr{I}_{0+}^{\lambda} f$ (e.g., [7]):

$$
\left(\mathscr{P}_{0+}^{\lambda-1,0} f\right)(x)=\Gamma(\lambda)\left(\mathscr{I}_{0+}^{\lambda} f\right)(x), \quad(\Re(\lambda)>1) .
$$

PFI operator (7) leads to numerous interesting illustrations such as fractional calculus associated with probability density functions and their significant in statistical theory. Nowadays, many researchers study PFI formulae associated with various special functions (see [18-27]). Motivated by these researchers, we study the $\mathcal{S}$-function, which is connected with PFI operator (7), to present their integral formulae. Suitable connections of some particular cases are also pointed out.

\section{Pathway Fractional Integral Operator of $\delta$ - Function}

In this section, we establish the PFI formula involving the $\mathcal{S}$-function which is stated in Theorems 1 and 2 .

Theorem 1. Suppose $w, k \in \mathfrak{R}, \sigma, \eta, \varepsilon, \tau \in \mathbb{C}, \mathfrak{R}(\sigma)>0, \mathfrak{R}(\lambda)$ $>0, \mathfrak{R}(\sigma)>k \Re(\tau)$, and $p<q+1, \mathfrak{R}(\lambda /(1-\varsigma))>-1 ; \varsigma<1$. Then, the following formula holds true:

$$
\begin{aligned}
& \mathscr{P}_{0^{+}}^{\lambda, \varsigma}\left[\zeta^{(\eta / k)-1} \mathcal{S}_{(p, q)}^{\sigma, \eta, \varepsilon, \tau, k}\left[l_{1}, l_{2}, \ldots, l_{p} ; m_{1}, m_{2}, \ldots, m_{q} ; w \zeta^{(\sigma / k)}\right]\right](x) \\
& =\frac{x^{\lambda+(\eta / k)} k^{(1+(\lambda / 1-\varsigma))} \Gamma(\lambda /(1-\varsigma)+1)}{(a(1-\varsigma))^{(\eta / k)}} \times \mathcal{S}_{(p, q)}^{\sigma, \eta+(1+\lambda /(1-\varsigma)) k, \varepsilon, \tau, k}\left[l_{1}, l_{2}, \ldots, l_{p} ; m_{1}, m_{2}, \ldots, m_{q} ; \frac{w x^{(\sigma / k)}}{(a(1-\varsigma))^{(\sigma / k)}}\right] .
\end{aligned}
$$

Proof. We indicate the RHS of equation (13) by $\mathfrak{\Im}_{1}$, and invoking equations (1) and (7), we have

$$
\begin{aligned}
\mathfrak{J}_{1}= & x^{\lambda} \int_{0}^{[x / a(1-\varsigma)]}\left(1-\frac{a(1-\varsigma) \zeta}{x}\right)^{\lambda /(1-\varsigma)} \zeta^{(\eta / k)-1} \\
& \times \sum_{n=0}^{\infty} \frac{\left(l_{1}\right)_{n} \ldots\left(l_{p}\right)_{n}(\varepsilon)_{n \tau, k}}{\left(m_{1}\right)_{n} \ldots\left(m_{q}\right)_{n} \Gamma_{k}(n \sigma+\eta)} \frac{\left(w \zeta^{(\sigma / k)}\right)^{n}}{n !} \mathrm{d} \zeta .
\end{aligned}
$$

Now changing the order of integration and summation, we obtain

$$
\begin{aligned}
\mathfrak{J}_{1}= & x^{\lambda} \sum_{n=0}^{\infty} \frac{\left(l_{1}\right)_{n} \ldots\left(l_{p}\right)_{n}(\varepsilon)_{n \tau, k} w^{n}}{\left(m_{1}\right)_{n} \ldots\left(m_{q}\right)_{n} \Gamma_{k}(n \sigma+\eta) n !} \\
& \times \int_{0}^{[x / a(1-\varsigma)]}\left(1-\frac{a(1-\varsigma) \zeta}{x}\right)^{\lambda /(1-\varsigma)} \zeta^{((\eta+\sigma n) / k)-1} \mathrm{~d} \zeta .
\end{aligned}
$$

Using the substitution $u=a(1-\varsigma) \zeta / x$, we can change the limit of integration into the following:

$$
\begin{aligned}
\mathfrak{\Im}_{1}= & x^{\lambda} \sum_{n=0}^{\infty} \frac{\left(l_{1}\right)_{n} \ldots\left(l_{p}\right)_{n}(\varepsilon)_{n \tau, k} w^{n}}{\left(m_{1}\right)_{n} \ldots\left(m_{q}\right)_{n} \Gamma_{k}(n \sigma+\eta) n !}\left(\frac{x}{a(1-\varsigma)}\right)^{(\eta+\sigma n) / k} \\
& \times \int_{0}^{1}(1-u)^{\lambda /(1-\varsigma)} u^{((\eta+\sigma n) / k)-1} \mathrm{~d} u .
\end{aligned}
$$

Now, by calculating the inner integral and using the beta function formula, we obtain the following:

$$
\begin{aligned}
\mathfrak{\Im}_{1}= & x^{\lambda} \sum_{n=0}^{\infty} \frac{\left(l_{1}\right)_{n} \ldots\left(l_{p}\right)_{n}(\varepsilon)_{n \tau, k} w^{n}}{\left(m_{1}\right)_{n} \ldots\left(m_{q}\right)_{n} \Gamma_{k}(n \sigma+\eta) n !}\left(\frac{x}{a(1-\varsigma)}\right)^{(\eta+\sigma n) / k} \\
& \times \frac{\Gamma(\eta / k+n \sigma / k) \Gamma(\lambda /(1-\varsigma)+1)}{\Gamma(\eta / k+(n \sigma / k)+\lambda /(1-\varsigma)+1)} .
\end{aligned}
$$

Using (3), we obtain 


$$
\begin{aligned}
\mathfrak{I}_{1}= & x^{\lambda+\eta / k} \sum_{n=0}^{\infty} \frac{\left(l_{1}\right)_{n} \ldots\left(l_{p}\right)_{n}(\varepsilon)_{n \tau, k}}{\left(m_{1}\right)_{n} \ldots\left(m_{q}\right)_{n} \Gamma(n \sigma / k+\eta / k) k^{(n \sigma / k)+(\eta / k)-1} n !} \\
& \times \frac{\Gamma(\eta / k+n \sigma / k) \Gamma(\lambda /(1-\varsigma)+1)}{(a(1-\varsigma))^{\eta / k} \Gamma(\eta / k+(n \sigma / k)+\lambda /(1-\varsigma)+1)} \\
& \cdot\left(w\left(\frac{x}{a(1-\varsigma)}\right)^{(\sigma) / k}\right)^{n} .
\end{aligned}
$$

Once again, using (3), we obtain

$$
\begin{aligned}
\mathfrak{I}_{1}= & \frac{x^{\lambda+(\eta / k)} k^{(1+\lambda /(1-\varsigma))} \Gamma(\lambda /(1-\varsigma)+1)}{(a(1-\varsigma))^{(\eta / k)}} \\
& \delta_{(p, q)}^{\sigma, \eta+(1+\lambda /(1-\varsigma)) k, \varepsilon, \tau, k}\left[l_{1}, l_{2}, \ldots, l_{p} ; m_{1}, m_{2}, \ldots,\right. \\
& \left.m_{q} ; \frac{w x^{(\sigma / k)}}{(a(1-\varsigma))^{(\sigma / k)}}\right]
\end{aligned}
$$

which gives the required proof of Theorem 1 .

Corollary 1. If we put $p=q=0$, then (13) leads to the subsequent result of generalized k-Mittag-Leffler function:

$$
\begin{aligned}
\mathscr{P}_{0^{+}}^{\lambda, \varsigma} & {\left[\zeta^{(\eta / k)-1} \mathscr{E}_{k, \sigma, \eta}^{\mathcal{\varepsilon}, \tau}\left(w \zeta^{(\sigma / k)}\right)\right](x) } \\
= & \frac{x^{\lambda+(\eta / k)} k^{(1+\lambda /(1-\varsigma))} \Gamma(\lambda /(1-\varsigma)+1)_{\mathscr{E}_{k, \sigma, \eta+(1+\lambda /(1-\varsigma)) k}^{\mathcal{,},}}(x)}{(a(1-\varsigma))^{(\eta / k)}} \\
& \cdot\left[\frac{w x^{(\sigma / k)}}{(a(1-\varsigma))^{(\sigma / k)}}\right]
\end{aligned}
$$

Proof. We consider (4) and $p=q=0$ in Theorem 1, and we obtain the desired result in (13).

Corollary 2. If we put $k=\tau=1$, then (13) leads to the subsequent result in terms of generalized $\mathscr{K}$-function:

$$
\begin{aligned}
\mathscr{P}_{0^{+}}^{\lambda, \zeta} & {\left[\zeta^{\eta-1} \mathscr{K}_{(p, q)}^{\sigma, \eta, \mathcal{\varepsilon}}\left[l_{1}, l_{2}, \ldots, l_{p} ; m_{1}, m_{2}, \ldots, m_{q} ; w \zeta^{\sigma}\right]\right](x) } \\
= & \frac{x^{\lambda+\eta} \Gamma(\lambda /(1-\varsigma)+1)}{(a(1-\varsigma))^{\eta}} \mathscr{K}_{(p, q)}^{\sigma, \eta+(1+\lambda /(1-\varsigma)), \varepsilon} \\
& \cdot\left[l_{1}, l_{2}, \ldots, l_{p} ; m_{1}, m_{2}, \ldots, m_{q} ; \frac{w x^{\sigma}}{(a(1-\varsigma))^{\sigma}}\right] .
\end{aligned}
$$

Proof. If we set $k=\tau=1$ in Theorem 1 and using (5), we obtain the required result (21).

Corollary 3. If we put $k=\tau=1$, then (13) holds the formula in terms of generalized $\mathscr{M}$-series:

$$
\begin{aligned}
\mathscr{P}_{0^{+}}^{\lambda, \varsigma} & {\left[\zeta^{\eta-1} \mathscr{M}_{(p, q)}^{\sigma, \eta}\left[l_{1}, l_{2}, \ldots, l_{p} ; m_{1}, m_{2}, \ldots, m_{q} ; w \zeta^{\sigma}\right]\right](x) } \\
& =\frac{x^{\lambda+\eta} \Gamma(\lambda /(1-\varsigma)+1)}{(a(1-\varsigma))^{\eta}} \mathscr{M}_{(p, q)}^{\sigma, \eta+(1+\lambda /(1-\varsigma))} \\
& \cdot\left[l_{1}, l_{2}, \ldots, l_{p} ; m_{1}, m_{2}, \ldots, m_{q} ; \frac{w x^{\sigma}}{(a(1-\varsigma))^{\sigma}}\right]
\end{aligned}
$$

Proof. If we put $\tau=k=\varepsilon=1$ in Theorem 1 and using (6), we obtain the result (22).

Now, we use equation (10) to define the following theorem, by the case $\varsigma>1$.

Theorem 2. Suppose $w, k \in \mathfrak{R} ; \sigma, \eta, \varepsilon, \tau \in \mathbb{C}, \mathfrak{R}(\sigma)>0$, $\mathfrak{R}(\lambda)>0, \mathfrak{R}(\sigma)>k \mathfrak{R}(\tau) \quad$ and $\quad p<q+1, \quad$ and $\mathfrak{R}(1-(\lambda / \varsigma-1))>0 ; \varsigma>1$. Then, the following formula holds true:

$$
\begin{aligned}
\mathscr{P}_{0^{+}}^{\lambda, \varsigma}\left[\zeta^{(\eta / k)-1} \mathcal{S}_{(p, q)}^{\sigma, \eta, \tau, \tau, k}\left[l_{1}, l_{2}, \ldots, l_{p} ; m_{1}, m_{2}, \ldots, m_{q} ; w \zeta^{(\sigma / k)}\right]\right] \\
(x)= \\
\quad \times \frac{x^{\lambda+(\eta / k)} k^{(1-\lambda /(\varsigma-1))} \Gamma(1-(\lambda /(\varsigma-1)))}{(-a(\varsigma-1))^{(\eta / k)}} \\
\quad \mathcal{S}_{(p, q)}^{\sigma, \eta+(1-\lambda /(\varsigma-1)) k, \varepsilon, \tau, k}\left[l_{1}, l_{2}, \ldots, l_{p} ; m_{1}, m_{2}, \ldots, m_{q} ;\right. \\
\\
\left.\quad \frac{w x^{(\sigma / k)}}{(-a(\varsigma-1))^{(\sigma / k)}}\right] .
\end{aligned}
$$

Proof. We denote, for convenience, the RHS of equation (23) by $\mathfrak{I}_{2}$, and invoking equations (1) and (10), we have

$$
\begin{aligned}
\mathfrak{J}_{2}= & x^{\lambda} \int_{0}^{[x /-a(\varsigma-1)]}\left(1+\frac{a(\varsigma-1) \zeta}{x}\right)^{\lambda /-(\varsigma-1)} \zeta^{(\eta / k)-1} \\
& \times \sum_{n=0}^{\infty} \frac{\left(l_{1}\right)_{n} \ldots\left(l_{p}\right)_{n}(\varepsilon)_{n \tau, k}}{\left(m_{1}\right)_{n} \ldots\left(m_{q}\right)_{n} \Gamma_{k}(n \sigma+\eta)} \frac{\left(w \zeta^{(\sigma / k)}\right)^{n}}{n !} \mathrm{d} \zeta .
\end{aligned}
$$

Now, changing the order of integration and summation, we obtain 


$$
\begin{aligned}
\mathfrak{J}_{2}= & x^{\lambda} \sum_{n=0}^{\infty} \frac{\left(l_{1}\right)_{n} \ldots\left(l_{p}\right)_{n}(\varepsilon)_{n \tau, k} w^{n}}{\left(m_{2}\right)_{n} \ldots\left(m_{q}\right)_{n} \Gamma_{k}(n \sigma+\eta) n !} \\
& \times \int_{0}^{[x /-a(\varsigma-1)]}\left(1+\frac{a(\varsigma-1) \zeta}{x}\right)^{\lambda /-(\varsigma-1)} \zeta^{((\eta+\sigma n) / k)-1} \mathrm{~d} \zeta .
\end{aligned}
$$

By setting $v=-a(\varsigma-1) \zeta / x$, we can change the limit of integration into the following:

$$
\begin{aligned}
\mathfrak{I}_{2}= & x^{\lambda} \sum_{n=0}^{\infty} \frac{\left(l_{1}\right)_{n} \ldots\left(l_{p}\right)_{n}(\varepsilon)_{n \tau, k} w^{n}}{\left(m_{1}\right)_{n} \ldots\left(m_{q}\right)_{n} \Gamma_{k}(n \sigma+\eta) n !}\left(\frac{x}{-a(\varsigma-1)}\right)^{(\eta+\sigma n) / k} \\
& \times \int_{0}^{1}(1-v)^{\lambda /-(\varsigma-1)} v^{((\eta+\sigma n) / k)-1} \mathrm{~d} v \\
& \times \int_{0}^{1}(1-v)^{\lambda /-(\varsigma-1)} v^{((\eta+\sigma n) / k)-1} \mathrm{~d} v .
\end{aligned}
$$

By analyzing the internal integral and using the beta function rule, we obtain

$$
\begin{aligned}
\mathfrak{\Im}_{2}= & x^{\lambda} \sum_{n=0}^{\infty} \frac{\left(l_{1}\right)_{n} \ldots\left(l_{p}\right)_{n}(\varepsilon)_{n \tau, k} w^{n}}{\left(m_{1}\right)_{n} \ldots\left(m_{q}\right)_{n} \Gamma_{k}(n \sigma+\eta) n !}\left(\frac{x}{-a(\varsigma-1)}\right)^{(\eta+\sigma n) / k} \\
& \times \frac{\Gamma(\eta / k+n \sigma / k) \Gamma(1-\lambda /(\varsigma-1))}{\Gamma(\eta / k+(n \sigma / k)+1-\lambda /(\varsigma-1))} .
\end{aligned}
$$

Using (3), we obtain

$$
\begin{aligned}
\mathfrak{\Im}_{2}= & x^{\lambda+\eta / k} \sum_{n=0}^{\infty} \frac{\left(l_{1}\right)_{n} \ldots\left(l_{p}\right)_{n}(\varepsilon)_{n \tau, k}}{\left(m_{1}\right)_{n} \ldots\left(m_{q}\right)_{n} \Gamma(n \sigma / k+\eta / k) k^{(n \sigma / k)+(\eta / k)-1} n !} \\
& \times \frac{\Gamma(\eta / k+n \sigma / k) \Gamma(1-\lambda /(\varsigma-1))}{(-a(\varsigma-1))^{\eta / k} \Gamma(\eta / k+(n \sigma / k)+1-\lambda /(\varsigma-1))} \\
& \cdot\left(w\left(\frac{x}{-a(\varsigma-1)}\right)^{(\sigma) / k}\right)^{n} .
\end{aligned}
$$
(3):

Once again, we arrive at the target outcome by applying

$$
\begin{aligned}
\mathfrak{I}_{2}= & \frac{x^{\lambda+(\eta / k)} k^{(1-\lambda / \varsigma-1)} \Gamma(1-\lambda / \varsigma-1)}{(-a(\varsigma-1))^{(\eta / k)}} \mathcal{S}_{(p, q)}^{\sigma, \eta+(1-\lambda / \varsigma-1) k, \mathcal{\varepsilon}, \tau, k} \\
& \cdot\left[l_{1}, l_{2}, \ldots, l_{p} ; m_{1}, m_{2}, \ldots, m_{q} ; \frac{w x^{(\sigma / k)}}{(-a(\varsigma-1))^{(\sigma / k)}}\right] .
\end{aligned}
$$

Corollary 4. If we put $p=q=0$, then (23) provides the result as follows:

$$
\begin{aligned}
& \mathscr{P}_{0^{+}}^{\lambda, \zeta}\left[\zeta^{(\eta / k)-1} \mathscr{E}_{k, \sigma, \eta}^{\mathcal{E}, \tau}\left(w \zeta^{(\sigma / k)}\right)\right](x) \\
& =\frac{x^{\lambda+(\eta / k)} k^{(1-(\lambda / \varsigma-1))} \Gamma(1-(\lambda / \varsigma-1))}{(-a(\varsigma-1))^{(\eta / k)}} \mathscr{E}_{k, \sigma, \eta+(1-(\lambda / \varsigma-1)) k}^{\mathcal{\varepsilon}, \tau}(x) \\
& \cdot\left[\frac{w x^{(\sigma / k)}}{(-a(\varsigma-1))^{(\sigma / k)}}\right] \text {. } \\
& \mathscr{P}_{0^{+}}^{\lambda, \zeta}\left[\zeta^{\eta-1} \mathscr{K}_{(p, q)}^{\sigma, \eta, \varepsilon}\left[l_{1}, l_{2}, \ldots, l_{p} ; m_{1}, m_{2}, \ldots, m_{q} ; w \zeta^{\sigma}\right]\right](x) \\
& =\frac{x^{\lambda+\eta} \Gamma(1-(\lambda / \varsigma-1))}{(-a(\varsigma-1))^{\eta}} \mathscr{K}_{(p, q)}^{\sigma, \eta+(1-(\lambda / \varsigma-1)), \varepsilon} \\
& \cdot\left[l_{1}, l_{2}, \ldots, l_{p} ; m_{1}, m_{2}, \ldots, m_{q} ; \frac{w x^{\sigma}}{(-a(\varsigma-1))^{\sigma}}\right] .
\end{aligned}
$$

Proof. If we set $k=\tau=1$ in Theorem 2 and using (5), we obtain the required result (31).

Corollary 6. If we put $k=\tau=\varepsilon=1$, then resulting formula (23) holds true:

$$
\begin{aligned}
\mathscr{P}_{0^{+}}^{\lambda, \varsigma} & {\left[\zeta^{\eta-1} \mathscr{M}_{(p, q)}^{\sigma, \eta}\left[l_{1}, l_{2}, \ldots, l_{p} ; m_{1}, m_{2}, \ldots, m_{q} ; w \zeta^{\sigma}\right]\right](x) } \\
= & \frac{x^{\lambda+\eta} \Gamma(1-(\lambda / \varsigma-1))}{(-a(\varsigma-1))^{\eta}} \mathscr{M}_{(p, q)}^{\sigma, \eta+(1-(\lambda / \varsigma-1))} \\
& \cdot\left[l_{1}, l_{2}, \ldots, l_{p} ; m_{1}, m_{2}, \ldots, m_{q} ; \frac{w x^{\sigma}}{(-a(\varsigma-1))^{\sigma}}\right]
\end{aligned}
$$

Proof. If we put $\tau=k=\varepsilon=1$ in Theorem 2 and using (6), we obtain the result (32).

\section{Concluding Remarks}

In the present paper, we have established two pathway fractional integral formulae associated with the more generalized special function called as S-function. The results 
obtained here involve special functions such as $k$-Mittag-Leffler function, $\mathscr{K}$-function, and $\mathscr{M}$-series, due to their general nature and usefulness in the theory of integral operators and relevant part of computational mathematics. Also, the special functions involved here can be reduced to simpler functions, which have a number of applications in various fields of science and technology and can be found as special cases that we have not specifically stated here.

\section{Data Availability}

No data were used to support this study.

\section{Conflicts of Interest}

The authors declare that they have no conflicts of interest.

\section{Authors' Contributions}

All authors contributed equally to the present investigation and read and approved the final manuscript.

\section{References}

[1] V. S. Kiryakova, "Generalized fractional calculus and applications," in Pitman Research Notes in Mathematics Series, Wiley, New York, NY, USA, 1993.

[2] K. S. Miller and B. Ross, An Introduction to the Fractional Calculus and Fractional Differential Equations, A WileyInterscience Publication, New York, NY, USA, 1993.

[3] R. K. Saxena and J. Daiya, "Integral transforms of S-functions," Matematiche (Catania), vol. 70, no. 2, pp. 147-159, 2015.

[4] D. L. Suthar and H. Amsalu, "Fractional integral and derivative formulas by using Marichev-Saigo-Maeda operators involving the S-function," Abstract and Applied Analysis, vol. 2019, Article ID 6487687, 19 pages, 2019.

[5] R. Díaz and E. Pariguan, "On hypergeometric functions and Pochhammer k-symbol," Divulgaciones Matematicas, vol. 15, no. 2, pp. 179-192, 2007.

[6] L. Romero and R. Cerutti, "Fractional fourier transform and special $k$-function," International Journal of Contemporary Mathematical Sciences, vol. 7, no. 4, pp. 693-704, 2012.

[7] R. K. Saxena, J. Daiya, and A. Singh, "Integral transforms of the $k$-generalized Mittag-Leffler function," Matematiche (Catania), vol. 69, no. 2, pp. 7-16, 2014.

[8] H. Amsalu and D. L. Suthar, "Generalized fractional integral operators involving Mittag-Leffler function," Abstract and Applied Analysis, vol. 2018, Article ID 7034124, 8 pages, 2018.

[9] S. D. Purohit, S. L. Kalla, and D. L. Suthar, "Fractional integral operators and the multiindex Mittag-Leffler functions," Scientia Series A: Mathematical Sciences, vol. 21, pp. 87-96, 2011.

[10] K. Sharma, "Application of fractional calculus operators to related area," General Mathematics Notes, vol. 7, no. 1, pp. 33-40, 2011.

[11] D. L. Suthar and H. Habenom, "Marichev-saigo integral operators involving the product of $\mathrm{K}$-function and multivariable polynomials," Communications in Numerical Analysis, vol. 2017, no. 2, pp. 101-108, 2017.

[12] M. Sharma and R. Jain, "A note on a generalized M-series as a special function of fractional calculus," Fractional Calculus and Applied Analysis, vol. 12, no. 4, pp. 449-452, 2009.

[13] S. D. Purohit, D. L. Suthar, and S. L. Kalla, "Some results on fractional calculus operators associated with the M-function," Hadronic Journal, vol. 33, no. 3, pp. 225-235, 2010.

[14] S. S. Nair, "Pathway fractional integration operator," Fractional Calculus and Applied Analysis, vol. 12, pp. 237-252, 2009.

[15] A. M. Mathai, "A pathway to matrix-variate gamma and normal densities," Linear Algebra and Its Applications, vol. 396, pp. 317-328, 2005.

[16] A. M. Mathai and H. J. Haubold, "Pathway model, superstatistics, Tsallis statistics, and a generalized measure of entropy," Physica A: Statistical Mechanics and Its Applications, vol. 375 , no. 1, pp. 110-122, 2007.

[17] A. M. Mathai and H. J. Haubold, "On generalized distributions and pathways," Physics Letters A, vol. 372, no. 12, pp. 2109-2113, 2008.

[18] P. Agarwal and J. Choi, "Certain fractional integral inequalities associated with pathway fractional integral operators," Bulletin of the Korean Mathematical Society, vol. 53, no. 1, pp. 181-193, 2016.

[19] P. Agarwal and S. D. Purohit, "The unified pathway fractional integral formulae," Journal of Fractional Calculus and Applications, vol. 4, no. 1, pp. 105-112, 2013.

[20] D. Baleanu and P. Agarwal, "A composition formula of the Pathway integral transform operator," Note di Matematica, vol. 34, no. 2, pp. 145-155, 2014.

[21] J. Choi and P. Agarwal, "Certain inequalities involving pathway fractional integral operators," Kyungpook Mathematical Journal, vol. 56, no. 4, pp. 1161-1168, 2016.

[22] S. Jain, P. Agarwal, and A. Kilicman, "Pathway fractional integral operator associated with $3 \mathrm{~m}$-parametric MittagLeffler functions," International Journal of Applied and Computational Mathematics, vol. 4, p. 115, 2018.

[23] D. Kumar, R. K. Saxena, and J. Daiya, "Pathway fractional integral operators of generalized k-wright function and k4function," Boletim da Sociedade Paranaense de Matemática, vol. 35, no. 2, pp. 235-246, 2017.

[24] K. S. Nisar, S. R. Mondal, and P. Agarwal, "Pathway fractional integral operator associated with Struve function of first kind," Advanced Studies in Contemporary Mathematics (Kyungshang), vol. 26, pp. 63-70, 2016.

[25] K. S. Nisar, S. D. Purohit, M. S. Abouzaid, M. Al-Qurashi, and D. Baleanu, "Generalized $k$-Mittag-Leffler function and its composition with pathway integral operators," Journal of Nonlinear Sciences and Applications, vol. 9, no. 6, pp. 35193526, 2016.

[26] G. Rahman, K. S. Nisar, J. Choi, S. Mubeen, and M. Arshad, "Pathway fractional integral formulas involving extended Mittag-Leffler functions in the kernel," Kyungpook Mathematical Journal, vol. 59, pp. 125-134, 2019.

[27] R. K. Saxena, J. Ram, and J. Daiya, "Fractional integration of the Aleph functions via pathway operator," International Journal of Physics and Mathematical Sciences, vol. 2, no. 1, pp. 163-172, 2012. 